\title{
Synchronous Optimization Design of Fixture Layout and Clamping Force Based on UG
}

\author{
Jin Fengfeng, Wang Guodong \\ Jiyuan Vocational and Technical college, Jiyuan459000,China
}

Keywords: fixture design; virtual simulation; machining process; UG software; clamping force

\begin{abstract}
In order to improve the accuracy and design efficiency of fixture, UG and ADMAS software has been introduced into the fixture design process, and the co-simulation function of software has been applied, and the fixture reasonable layout and the clamping force calculation and synchronous optimization has been realized. The fixture is carried out in the UG software, by using the machining process of the workpiece, and the fixture is fully opened by the addition of the motion constraint. The dynamics simulation is carried out by introducing UG fixture model into ADMAS software, and the sensitivity of the key points of the spring clamping force is obtained by calculating, the points that having greater impact on the spring clamping force is optimized, and the spring displacement of the variable point when the spring produces the maximum clamping force is obtained, so as to realize the fixture optimization design.
\end{abstract}

\section{Introduction}

In mechanical parts processing, the formation of parts size, surface relative position and the 3D geometry, mainly depends on the cutting tool and workpiece in machining process relative position relationship between the fixture is the tool constraints, workpiece and machine tool is the key link. Rational distribution and optimization of the fixture can guarantee the processing quality of the workpiece, improve productivity and expand the scope of the processing machine and clamp comprises clamping element, positioning component, a connecting component and other components, this study using CO simulation function of UG and ADAMS software, the fixture layout optimization, the clamping force of calculation and synchronous optimization, improve the precision and efficiency of fixture design.

\section{Workpiece machining process}

Fixture design and optimization mainly consists of the clamping force design and the overall design layout. Before the optimization design, you first need to determine the workpiece machining process, determine the process can make a reasonable layout of the fixture, clamping force of accounting, the workpiece as shown in Figure 1.

Figure 1 shows the schematic diagram of the processing of the workpiece, the process is mainly milling diameter of $32 \mathrm{~mm}$ hole end. The material of the workpiece is HT200, the material of the milling cutter is high speed steel, the diameter is $80 \mathrm{~mm}$, the number of teeth is 10 , we choose high speed steel cylindrical milling cutter diameter $d=60 \mathrm{~mm}$, tooth number $z=10$. Milling cutter shape, $r_{n}=10^{0}, a_{0}=12^{0}, \beta=45^{0}$, in the workpiece machining, the actual milling width $a_{e}=2.5 \mathrm{~mm}$, milling depth $a_{p}=50 \mathrm{~mm}$, we choose XA6132 horizontal milling machine. 


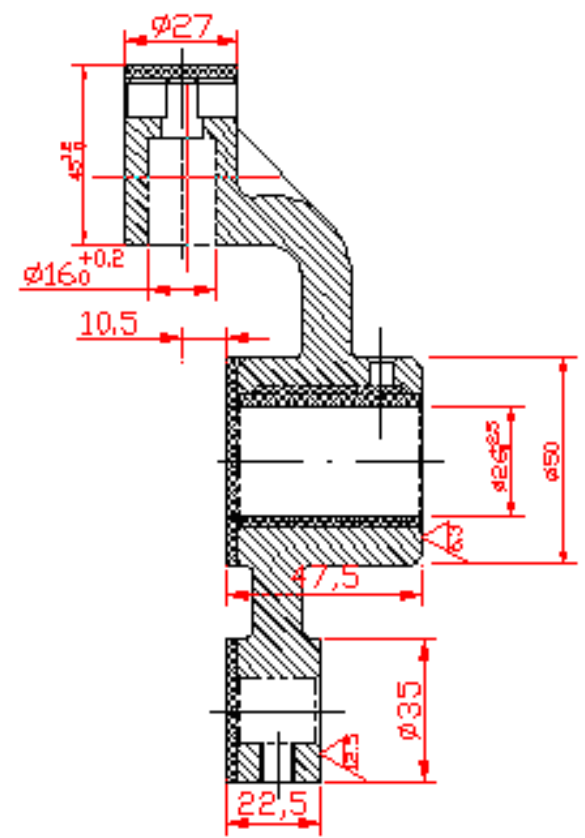

Fig. 1 Schematic diagram of machining workpiece

(1) Determine the feed per tooth $f_{\mathrm{z}}$

According to the machine power $7.5 \mathrm{~kW}$, the rigidity of the process system is medium, therefore, the feed rate of each tooth is $f_{\mathrm{z}}=0.16-0.24 \mathrm{~mm} / \mathrm{z}$, in this paper $f_{\mathrm{z}}=0.18 \mathrm{~mm} / \mathrm{z}$

(2) Selection of milling cutters standard

The criteria for selecting a milling cutter include wear and durability, according to the parameters of the process system, the data available, the maximum wear of the milling cutter is $1.5 \mathrm{~mm}$, the diameter of the milling cutter $d=60 \mathrm{~mm}$, and the durability of the milling cutter $T=180 \mathrm{~min}$.

(3) Determine the cutting speed

According to the above choice of milling parameters, we can get the actual speed of milling, and then according to the characteristics of XA6132 milling machine, check speed, $n_{\mathrm{c}}=300 \mathrm{r} / \mathrm{min}$, $V_{f c}=475 \mathrm{~mm} / \mathrm{s}$.

Actual cutting speed

$V_{c}=\frac{\pi d n_{c}}{1000}$

$V_{c}=\frac{3.14 \times 60 \times 300}{1000}=56.52 \mathrm{~m} / \mathrm{min}$

Actual feed rate

$$
\begin{aligned}
f_{z c} & =\frac{v_{f_{c}}}{n_{c} z} \\
f_{z c} & =\frac{475}{300 \times 10}=0.16 \mathrm{~mm} / \mathrm{z}
\end{aligned}
$$

According to the actual cutting speed and feed rate of the cutting tool, the fixture can be reasonably arranged so as to calculate the clamping force.

\section{UG fixture and layout design}

Fixture layout optimization by using the UG software, the first use of UG software to establish the simulation model of fixture, click the start button to enter the motion simulation module of UG nx6.0, 
the motion simulation of Navigation Centre right click and create a simulation. The gasket, the press block and the processing workpiece are defined as the three connecting rod structure, generally speaking, the connecting rod needs to have a fixed connecting rod, and the kinematic pair is fixed on the side, so the connecting rod is a living link.

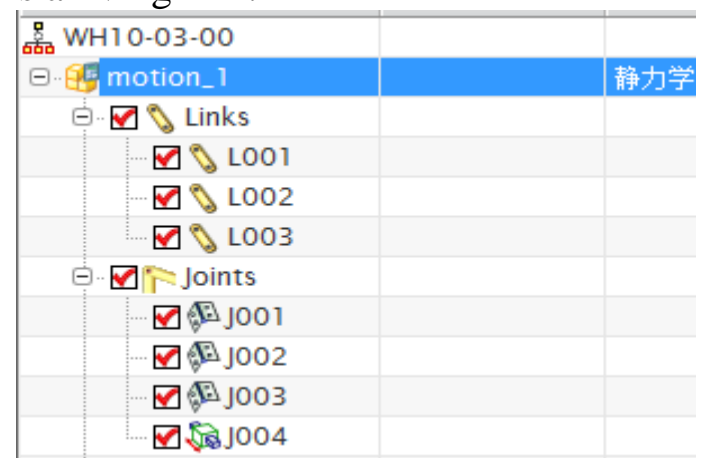

Fig. 2 positioning motion pairs and moving pairs

Figure 2 shows the movement and moving joints definition, in order to define the workpiece and the connection board, pressing plate arm and connecting plate, plate arm and the workpiece for three rotary side due to the connecting plate because there is no definition for the connecting rod, so the former two pairs of fixed. The drilling hole is set to move pair, and its motion direction is shown in Figure 3.

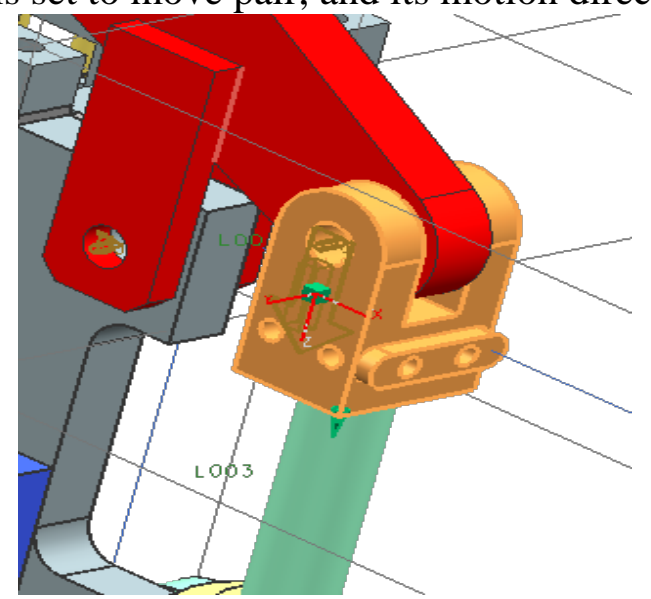

Fig. 3 the direction of movement of the set

Figure 3 shows mobile side direction diagram, in UG software, you can on the structure add secondary motion, and set the direction of movement pair, so as to drive the movement of the whole device, when the vice direction of movement can be in UG software fully open fixture, fully open status of fixture as shown in Figure 4. 


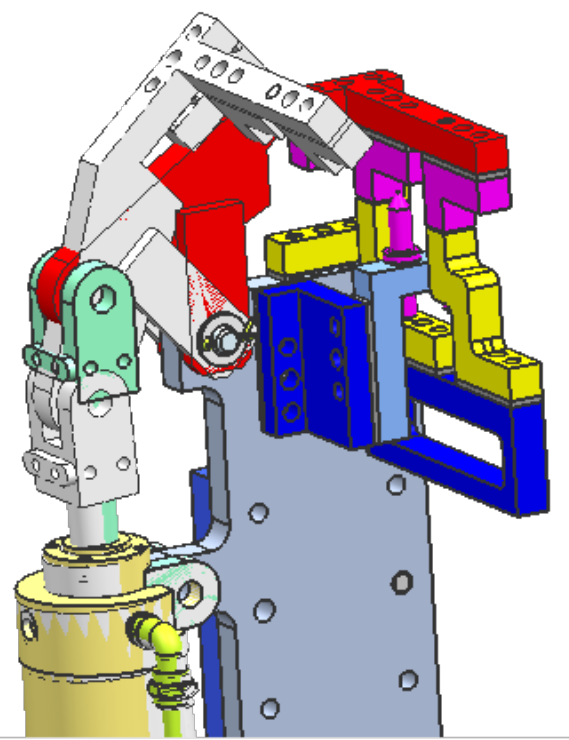

Fig.4 the fixture to complete the open state

Figure 4 shows fixture of the fully open state, the fixture is fully open and can simulate the workpiece clamping state. This is mainly to optimize the design of clamping force, clamping force optimization need based ADMAS virtual simulation to achieve.

\section{Clamping force simulation and synchronous optimization of fixture}

In order to achieve the simultaneous optimization of clamping force calculated clamping force size and requires a combination of ADMAS software, virtual simulation, ADMAS software and UG software compatibility is good, can be complex import UG model and the optimization steps are as follows:

(1) Thinning model

The main design points of the fixture to optimize the parameters of the fixture clamping mechanism to refine the different parameters of the clamping force.

(2) Iterative model

Through the optimization analysis function of ADAMS, find the position of the design point of the greatest influence on the clamping force.

(3) Optimal design

Get the maximum clamping force of several variables for the optimization of the variables, to obtain maximum clamping force.

In order to achieve the above steps, first in the ADMAS to open the variable optimization design of the dialog box, the main goal of optimization is to make the value of the spring force maximum, because it is negative, so take the minimum, as shown in figure 6 . 


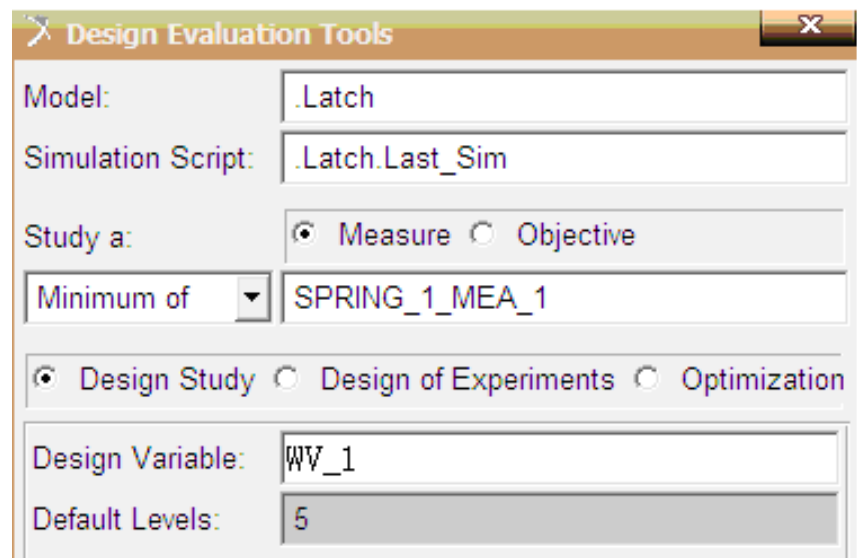

Fig.5 Variable optimization design dialog

Figure 5 represents the optimization design variables dialog box, point in a different location from the 10 optimization variables, variable names to WV 1. Main test of spring displacement variation, optimization design can be in ADMAS automatically generate reports, report content for the displacement of the spring of the initial value and the optimal value, as shown in table 1.

Table 1 Influence of design variables on the sensitivity of spring force

\begin{tabular}{cccc}
\hline Design variable name & Design point position & initial value/ $\mathrm{mm}$ & optimal value/ mm \\
\hline WV_1 & POINT_1x & 0 & 1.2 \\
WV_2 & POINT_1y & 0 & 1.1 \\
WV_3 & POINT_2x & 3 & 2.8 \\
WV_4 & POINT_2y & 3 & 3.1 \\
WV_5 & POINT_3x & 2 & 2.9 \\
WV_6 & POINT_3y & 8 & 9.2 \\
WV_7 & POINT_5x & -1 & -1.1 \\
WV_8 & POINT_5y & 10 & 10 \\
WV_9 & POINT_6x & -6 & -5.4 \\
WV_10 & POINT_6y & 5 & 4.5 \\
\hline
\end{tabular}

Table 1 shows the influence of design variables of the spring force sensitivity of simulation results, by table, it can be seen that WV3 design variables, 4, 7, 8 of the sensitivity of the initial value and the optimal value of the closest, most sensitive, the clamping force has the greatest impact.

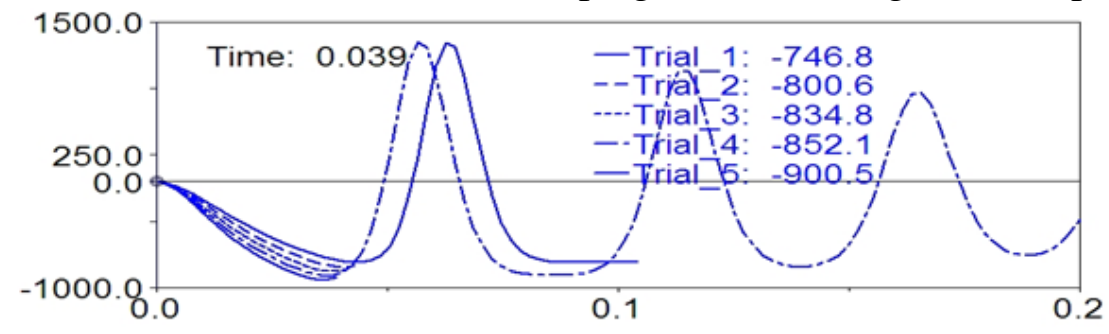

Fig.6 Spring clamping force optimization curve

Figure 6 is the spring clamping force optimization curve results, the figure can be seen, variable dv3, 4 , 7,8 values were $2.878,3.186,1.038,10.047$, clamping mechanism can generate maximum clamping force and maximum clamping force for $966.77 \mathrm{~N}$, so as to realize the optimization design of clamping force.

\section{Conclusion}

The layout of the fixture and clamping force were optimized to improve the design accuracy and efficiency of the fixture, by using UG software and ADMAS combined with virtual simulation in the 
paper. According to the process of fixture, in UG software established the complete fixture assembly, and the constrained motion features to add, so the fixture in the fully open state, will design a good fixture into ADMAS software, the fixture were dynamics simulation, the key variables affecting the magnitude of clamping force is obtained by simulation calculation. The key variables of the clamping force of the clamp are optimized, and the spring displacement of each variable point is obtained when the maximum spring clamping force is obtained.

\section{Reference}

[1] Zhou Xiaolun, Zhang Weihong, Qin Guohua, et al. Simultaneous optimization of fixture layout and clamping force based on genetic algorithm, mechanical science and technology, 2005, 24 (3): 339342

[2] Qin Guohua, Zhang Weihong, Zhou Xiaolun, mathematical modeling and clamping force optimization design, mechanical science and technology, mechanical science and technology, 2005, 24 (4): 438-442, 446

[3] Tian Shaopeng, Huang Zhengdong, Wu Sen, et al. Optimization method for the positioning and configuration of machining parts based on feature, tractor and agricultural transport vehicle, 2005, (4): 80-82

[4] Tian Shaopeng, Huang Zhengdong, Wu Sen, et al. The optimal design of the positioning and layout of the multi station shared installation, Journal of Wuhan University of Technology, 2005, 27 (8): 80-82

[5] Mei Zhongyi, Wang Yunqiao, Fan Yuqing, research and Simulation of NC machining deformation control of aircraft structure parts, aviation journal, 2005,26 (2): 234-239

[6] Wei Hua, Xu Jiuhua, Fu Yucan, et al. The optimization of hydrogen treatment process and hydrogen content of TC4 titanium alloy during the process of hydrogen cutting and processing China Mechanical Engineering, 2010,21 (2): 196-201.

[7] Hou Hongliang, Li Hong, Sun Zhonggang. The influence of hydrogen on the physical and mechanical properties of TC4 alloy and its correlation with the cutting performance. Aeronautical manufacturing technology, 2008, 20:80-88.

[8] Li Hong, Sun Zhonggang, Hou Hongliang, et al. Microstructure evolution and its influence on the chip formation of the hydrogen containing Ti_6Al_4V alloy. Mechanical science and technology, 2011,30 (3): 373-376.

[9] Cao Ziyang, He Ning, Li Liang. The formation and numerical simulation of high speed cutting titanium alloy Ti6Al4V chips. China Mechanical Engineering, 2008, 19 (20): 2450-2454.

[10] Wu Hongbing, Jia Zhixin, Liu Gang, et al. Finite element modeling of high speed machining of titanium alloy in high speed machining. Journal of Zhejiang University, 2010, 44 (5):982-987.

[11] Yang Zhenzhao, Zhang Dinghua, Yao Changfeng, et al. Effect of high speed milling on the surface integrity of TC4 titanium alloy. Journal of Nanjing University of Aeronautics \&amp; Astronautics, 2009, 41 (5): 644-648.

[12] Yang Shubao, Xu Jiuhua, Wei Hua, et al. Effects of hydrogen treatment on the rheological behavior of TC4 titanium alloy. Journal of Aeronautical Engineering, 2010,31 (5): 1093-1098. 